\title{
Simulation-based communication skills training for experienced clinicians to improve family conversations about organ and tissue donation
}

\section{Abstract}

Introduction: The approach, communication skills, and confidence of clinicians responsible for raising deceased organ donation may influence families' donation decisions.

The aim of this study was to increase the preparedness and confidence of intensive care clinicians allocated to work in a 'designated requester' role.

Design: We conducted a posttest evaluation of an innovative simulation-based training program. Simulation-based training enabled clinicians to rehearse the "balanced approach" to family donation conversations in the 'designated requester' role. Professional actors played family members in simulated clinical settings using authentic scenarios, with videoassisted reflective debriefing. Participants completed an evaluation after the workshop. Simple descriptive statistical analysis and content analysis were performed.

Results: Between January 2013 to July 2015, 25 workshops were undertaken with 86 participants; 82 (95.3\%) returned evaluations. Respondents were registered practicing clinicians; over half $(44 / 82 ; 53.7 \%)$ were intensivists. Most attended a single workshop. Evaluations were overwhelmingly positive with the majority rating workshops as outstanding $(64 / 80 ; 80 \%)$. Scenario fidelity, competence of the actors, opportunity to practice and receive feedback on performance, and feedback from actors, both in and out of character, were particularly valued. Most (76/78; 97.4\%) reported feeling more confident about their 'designated requester' role.

Discussion: Simulation-based communication training for the 'designated requester' role in family donation conversations increased the knowledge and confidence of clinicians to raise the topic of donation. 


\section{Background}

Conversations with potential organ donor families can be one of the most difficult clinical activities, irrespective of practitioner expertise or prior experience. Interactions playing out during these discussions can trigger raw emotions for loved ones and may influence opinions about organ donation. Recently, in Australia, approximately half of families approached to authorize organ donation on behalf of their relative have declined, a finding that contrasted with the high levels of support shown in population surveys. Evidence indicates that dedicated communication training focused on organ donation conversations increases health professionals' confidence and results in improved consent rates. ${ }^{1-3}$ Countries with high rates of organ donation such as Spain and the United States of America (USA), have used specialist requesters who completed specific donation conversation training. ${ }^{1,4,5}$ National reform strategies to increase organ donation rates included education for health professionals discussing organ donation with families. ${ }^{6}$ In 2011, the Professional Education Package (PEP) introduced an Australian "balanced approach" to help families in acute grief make organ donation decisions that were informed, proactive and enduring. ${ }^{7,8}$ The PEP modules provided opportunities to rehearse these conversations through role play with peers, and to practice answers to potential questions. ${ }^{7}$

Raising the topic of organ donation has historically been the responsibility of the intensivist managing care of the potential donor, with the donation specialist nurse (DSN) introduced to the family after they agreed to consider organ donation. ${ }^{9}$ In Australia education for this comprised attendance at a one-day donor awareness program that included communication training. ${ }^{10}$ Intensivists reported training was adequate preparation. ${ }^{11}$ Clinicians develop their approach and repertoire from observing colleagues' interactions with families, but opportunities to rehearse organ donation conversations occur only a few times per year for many intensivists. ${ }^{11}$ 
Health professionals have reported a tendency to avoid raising organ donation due to concerns about adding to a family's distress arising from their own perceptions of the emotional burden. ${ }^{12,13}$ It is unknown whether attending the PEP to train in the balanced approach is sufficient to enable experienced health professionals to confidently adopt the new process.

The designated requester (DR) role was introduced in New South Wales (NSW) in 2012. The DR was an experienced health professional who underwent specialized communication training to develop expertise to offer donation sensitively and improve decision making. ${ }^{14}$ This study aimed to evaluate the NSW Simulation Program of the family donation conversation (FDC) in relation to health care professionals' perceptions of its contribution to their preparedness and confidence to undertake the DR role.

\section{Methods}

\section{Design}

We conducted a posttest evaluation of an innovative simulation program with the specific aim of increasing the preparedness and confidence of clinicians undertaking the DR role.

\section{Setting}

The study was conducted in NSW. The provision of intensive care services is almost all exclusively the responsibility of accredited intensivists who manage all patients. Intensivists perform regular reviews where treatment goals/plans are adjusted according to patient needs. The registered nurse $(\mathrm{RN})$ to patient ratio is $1: 1$ for mechanically ventilated patients and RNs perform the majority of patient care.

The simulation program was conducted in simulation clinical laboratories equipped with full audio-visual (A-V) capabilities in a university health faculty in Sydney, Australia. The 
simulated intensive care unit (ICU) family meeting room was similar to a room available in most ICUs.

\section{Population}

Participants were eligible for this study if they were an experienced, practicing ICU clinician or donation specialist; had completed the PEP, and selection as a DR was confirmed by their ICU department head (or delegate). Invitations to participate in the simulation program were emailed from the OTDS.

\section{Intervention}

The simulation program was developed by a team of organ donation, intensive care medicine and simulation training experts. For practice-based professions meaningful learning is best if situated within authentic environments, is contextually based and incorporates interactions with peers and experts; concepts that lent themselves to simulation activities. ${ }^{15}$ The design incorporated educational strategies to increase learning: active participation and formative feedback, ${ }^{16}$ aligned with concepts from socio-material educational frameworks. ${ }^{17,18}$ Additional meaning was constructed through interactions between and with others, and with environmental materials (artifacts). The specific environment (for the FDC) was authentically represented in a simulation laboratory with arrangements of furniture, and the contextual materials critical for participant engagement were patient scenarios based on deidentified real cases (see Appendix). Interactions were planned with experts and peers (facilitators) and 'family members' portrayed by professional actors. Appropriately briefed, actors were able to realistically portray family member conversations and elicit meaningful engagement of participants resulting in socially constructed learning. ${ }^{17}$ Subsequent video-assisted and facilitated debriefing helped focus on 
specific areas and assisted with reflection and active co-construction or refinement of clinical practice. $^{19}$

Before the workshop, actors were provided a: debriefing guide; scenario synopsis; character outline including family background, personality, current state-of-mind; the level of emotional intensity expected could be varied in response to the participant. Actor briefing included the participant's experience level so they could tailor their questions and reactions. The actors prepared in a separate area to participants. Two weeks before the workshop participants received the program outline, workshop expectations, an assessment guide and a confidentiality agreement. At the beginning of the workshop, the facilitator reviewed this material with all participants. Those who took on the DR role in the simulation were advised to assume their usual work role (intensivist, DSN, or social worker), although few DSNs had initiated the topic of organ donation before. Participants were encouraged to take their time, to use the skills learned in the PEP, and not offer organ donation until they believed the family was ready.

\section{Procedures}

The three-part simulation workshop ran for four hours or a half-day. Each workshop catered for two participants; one enacted the role of requester while the other observed. These people swapped roles to experience or observe a different patient case scenario. Two simulation laboratories ran concurrently so that two half-day workshops accommodated eight participants per day.

Minimum personnel requirements for each workshop included two professional actors, one health care simulation expert (facilitator) and another subject expert. The subject expert often played the role of the bedside nurse. A minimum facility requirement was a simulated ICU family meeting room equipped with $\mathrm{A}-\mathrm{V}$ recording equipment and visual access for two 
observers: the facilitator, who made notes or annotated the recording while observing, and a participant observer (Box 1).

(Insert Box 1 about here)

The facilitator provided an overview of the patient scenario to the 'DR' and managing team. Each scenario commenced when the family had been informed either of the inevitable death or the death determined by neurological criteria ("brain death") of their loved one.

Part One: Planning meeting. The 'DR' participant met the managing intensivist and 'bedside nurse' to gather information and plan the FDC. They specified the manner of their introduction to the family; for example, either by stating they work in organ donation or by using general terms such as an "end-of-life specialist". A short debrief of this part followed (see Box 2 Part 1). Part Two: The FDC. The scenario and A-V recording began when the 'bedside nurse' showed the family into the simulated meeting room and joined the conversation. The 'intensivist' facilitator showed the 'DR' participant into the room and introduced the family in the manner determined in Part One, then left the room to observe and make notes. The participant led the FDC using the balanced approach, raising organ donation when appropriate. At the conclusion of the conversation the $\mathrm{A}-\mathrm{V}$ recording was stopped and a three-stage debriefing process was facilitated. ${ }^{19}$ The actors and nurse debriefed immediately, in and out of character (Box 2 Part 2). Part Three: Facilitated debriefing of the conversation. The video recorded conversation was viewed and discussed between the facilitator, subject expert, observer, and participant, guided by annotations or notes (Box 2 Part 3).

(Insert Box 2 about here)

Potential facilitator intervention and exit points were integrated into the simulations to ensure the workshops ran smoothly for 'DR' participants and family members. For example, if a 
participant felt the family needed a break and would normally divide the conversation, the participant drew the conversation to a temporary halt and left the room. The actors were briefed that time had elapsed, another meeting was scheduled and a second donation conversation was initiated from where the first was left. The simulated conversations were expected to take around an hour; the facilitator could intervene to bring it to a close if the conversation was not progressing.

\section{Data Collection}

Participants were invited to complete evaluation forms at the conclusion of each workshop. A Simulation Training Evaluation Form was created for the program (EM and MK), because there were no existing evaluation methods that aligned with this type of initiative. The form comprised eight items with five forced choice responses and three open-ended questions. Three items with "yes or no" response options and space for free text comments sought participants' views whether the workshop complemented or built on the PEP; its value as additional or essential training for the 'DR' role; if participants felt more confident undertaking the role after the workshop. Two items with Likert-scaled response options, from poor to outstanding recorded participants' expectations and overall opinions of the workshop.

Respondents were asked what they liked best and least, and suggestions for future developments.

\section{Data Analysis}

Quantitative and qualitative methods of analysis were used. Simple descriptive statistics (percentages and frequencies) reported quantitative data. Free text responses were transcribed verbatim and content analyzed for systematic interpretation. ${ }^{20}$ Two authors (experienced intensive care nurses: JP and RE) who were not involved in the development or delivery of the 
program performed the primary analysis. The items and responses were read repeatedly. Initially, common content within the responses was identified and coded using keywords. Similar or related words were confirmed in a thesaurus and grouped into categories manually by one researcher (RE) and using NVivo 10 for Windows (CQSR International) by another (JP). Responses were reread and the frequency that each category occurred was counted. Responses and keywords were reread some days after the initial content analysis to check for inconsistencies; none were found.

To reduce potential for bias, the analysts were blinded to respondents' designation and gender. To support credibility of the analysis, the selection of categories was identified independently and then discussed and agreed, with any disagreements settled in consultation with a third author (LP).

\section{Results}

Twenty-five simulation workshops were conducted between January 2013 and July 2015. Eighty-six health professionals were invited and participated, 82 (95.3\%) returned an evaluation form, with few incomplete responses. Respondents were practicing health professionals; more than half ( $\mathrm{n}=44 ; 53.7 \%)$ were intensivists (denoted as ' $\mathrm{M}$ '; nurses as ' $\mathrm{N}$ '; social workers as 'SW') (Table 1). The majority attended a single workshop.

(Insert Table 1 about here)

All workshops were delivered without any participant withdrawing from any component. The simulated donation conversations lasted on average 40 minutes; most were effectively managed by the DRs with a few requiring facilitator input to bring the conversation to a timely close. 
Quantitative evaluation was overwhelmingly positive. The respondents rated the simulation workshop highly $(78 / 81 ; 96.3 \%)$ and agreed that it complemented and built on the PEP. Nearly all $(78 / 79 ; 98.7 \%)$ agreed that it was valuable or necessary training for the DR role. Most $(76 / 78 ; 97.4 \%)$ subsequently felt more confident to be a DR. Expectations of the training were well met, and rated as outstanding $(63 / 80 ; 78.8 \%)$ or good $(17 / 80 ; 21.3 \%)$.

Qualitative evaluation was predominantly positive. Three respondents disagreed that the simulation workshop built on national training but commented that it “...stands alone in its own right" (M), and "may be helpful for someone starting out but not for experienced clinicians" (M). Personal insights from the simulation workshop included that:

...It is possible that the CORE and Practical sessions affected my practice in some ways. I think I will be more self-conscious of my performance after the simulation session (M).

Three respondents who did not respond to the value of simulation training commented positively (two respondents) and the other stated a preference for mentorship by experienced clinicians: "A single session in isolation is interesting, but a larger group forum with senior colleagues would be more useful" (M). One respondent, whose confidence did not improve, felt they had learnt a lot about communication.

All respondents gave examples of what they liked most. The main categories identified were feedback, use of professional actors, and realism (Table 2).

(Insert Table 2 about here)

For the 'feedback' category the value of the actors' feedback was specifically highlighted: "The FB [sic] from the actors both in character and out of character was exceptional" (M \& N). The quality of feedback overall was rated as good to excellent: "Good feedback, very helpful debriefing sessions and ability to relate to peers" (M). Respondents 
valued this feedback because of their respect for the experts providing it: "Excellent feedback from experienced educators" (M). The topics covered included key elements of the national education, for example: “...the use of certain language or expansions" (M, SW). Participants appreciated the constructive manner in which it was delivered: "Peer debriefing was safe and constructive" $(\mathrm{N})$. Personal insights were gained from feedback with video playback: "Debrief with video. Really unnerving seeing myself in action. Will make me think" (M).

For the category for use of professional actors, their ability to portray an actual family's reactions, thereby immersing respondents in the unfolding scenario, was flagged: "The realistic scenario and how you forgot that they were in fact actors but a family going through this conversation" $(\mathrm{N})$; “The actors are exceptionally realistic. I had absolutely no problem engaging with them as if they were a real family. I enjoyed watching the scenarios of others" (M).

For the category of realism, the similarities of the scenarios to real situations was reiterated: "The scenarios were very realistic and the actors were very professional" (M); with tolerance for some loss of fidelity: “...not perfect but as close as you can get!” (M).

Other categories were opportunities to practice and the setting. Opportunities to practice mainly related to skill development: "Excellent realistic practice with good skill consolidation" (M). This allowed practice in responding to displays of emotion: "Realistic, with emotions and tears. Good to practice comments" (M). The workshop setting was a suitable place for learning, "Away from hospital, well set out and well managed as a collegial non-threatening exercise" (M). Seven respondents made similar comments.

Most respondents (66/82; 80.5\%) detailed what they liked least (Table 3). For many this was nothing, reflecting the overall positive evaluation. Performance anxiety, being watched, and the setting were raised. 
(Insert Table 3 about here)

Performance anxiety occurred before and during the workshop: "Anxiety and apprehension on my part before participation” $(\mathrm{M}) ;$ “... people around you and observing you is nerve racking” $(\mathrm{N})$. Many disliked watching themselves on video. The inconvenience of locating the workshop in the city and the room design were raised. Personal reactions to criticism were highlighted: "I don't take criticism well, but I understand and value it" (M). Finally, the unpredictability of actor reactions was raised and a few participants had difficulty engaging in the observer role.

Fifty-nine participants (72\%) made suggestions for future developments. Half related to providing more sessions such as annual refreshers or repeat attendance. More difficult scenarios that included family disagreement, pediatric cases, and donation after circulatory determination of death were also suggested. Feedback suggestions included providing real-time feedback from a facilitator while watching the alternate scenario and creating a montage of the best aspects discussed at some of the simulations.

\section{Discussion}

We developed this simulation program to prepare intensivists, DSNs, and social workers involved with donation conversations to undertake the DR role. Participants' evaluations were overwhelmingly positive with most agreeing the program was valuable, complemented the PEP and increased their confidence as a DR. Debriefing with actors in and out of character was viewed as powerful and a rare opportunity for appraisal of one's performance during an emotional conversation with a family member in acute grief. The video-reflexive feedback was especially useful in identifying areas for improvement in requesters' body language,

phraseology, and pace of conversation. Aspects least liked related to performance anxiety, the 
observer role, and being observed. These results support the use of simulation training to increase the preparedness of experienced clinicians as DRs.

This study examined participants' opinions of rehearsing the donation conversation using the balanced approach in actual, deidentified potential organ donor scenarios with a comprehensive debriefing process. Other programs have also used feedback from peers and facilitators on performances in role-play of hypothetical scenarios with professional actors for communication training for multidisciplinary groups of clinicians exposed to difficult organ donation and end-of-life conversations in adult and pediatric populations previously. ${ }^{1,21-24}$ As in this study, participants reported simulation resulted in better preparation and greater confidence immediately posttraining and five-months later. ${ }^{23}$ ICU consultants showed improved sensitivity to relatives' needs when conveying news of death and raising organ donation at six months posttraining. ${ }^{24}$ Feedback from actors has been obtained using questionnaires or rating scales and sought directly during workshops and included in evaluations. ${ }^{21,22}$ In England, videotaped recordings of 64 ICU consultant-nurse pair encounters during hypothetical scenarios were independently rated. ${ }^{24}$ Videotaped recordings have also been used to highlight improvements in communication techniques in a group feedback session. ${ }^{21}$. A similar program, a one-day educational intervention on communicating about organ donation used real-time critique of videotaped performances of Organ Procurement Coordinator participants and additional expert debriefing of the simulation, has recently been reported. ${ }^{1}$ Key to the effectiveness of these feedback methods for learning was the creation of a safe environment.

Outcome measures have included consent rates for organ donation; an increase of $9.2 \%$ over two years after the intervention has been achieved. ${ }^{1}$ In Australia, organ donation consent rates have increased by $3 \%$ over two-years following introduction of the PEP. ${ }^{8}$ Participation in 
the simulation program, focusing on enhancing communication approaches combined with video-reflexive debriefing may increase the skills and preparedness of specialist requesters and further contribute to increased organ donation consent rates.

Participant evaluations of learning experiences of role modeling and the observer role have varied across jurisdictions. For example, in the European Donor Hospital Education Program workshops (United Kingdom), significantly higher learning was reported by those who actively role-played a doctor or a nurse compared to those who observed. This result contrasted with findings from the Netherlands where there was no difference in learning between those who role-played and those who observed. ${ }^{25}$ These differences may have been a result of experiences from previous training or requesting, or an effect of the level of participation ('dose') in scenarios. Preparation of the observer participants and how they contributed to the debriefing was discussed with a view to enhancing observer engagement during the simulation and debriefing. This is a growth area in simulation as the benefits of vicarious learning are clear and worth enhancing in the simulation program. ${ }^{26,27}$

The program had a number of strengths. The authenticity of the experience was universally appreciated; actors' expertise immersed participants in the scenarios. Real-life (deidentified) scenarios based on local case-mix, policies and procedures were fundamental to the authenticity. The simulation program was designed to enhance realism and maximize the time available to rehearse the FDC. The multidisciplinary training approximated the clinical environment and fostered collaboration between disciplines in support of bereaved families.

The program has some limitations. The workshops took place in a university simulation laboratory rather than in a clinical setting, and while the actors' performances were excellent, the realism of the surroundings was not perfect. Inevitably, the actors knew that organ donation 
would eventually be raised, and it was a challenge for some to 'reset' and remember specific dialogue in each scenario. Evaluation of simulation workshops using self-report questionnaires is open to subjectivity and bias. However, grasping a sense of the impact of the simulation on individuals' sense of professional practice is important for program evaluation, and this is an established, low-cost approach.

Evaluation of such programs is essential, both in the clinical environment and from the perspective of relatives, who ultimately are most affected by the quality of communication by health professionals. Furthermore factors affecting participation of experienced health professionals in communication skills training and adoption of alternative requesting approaches require investigation. The addition of a validated rating form such as the multi-rater communication skills instrument with gap analysis may increase the robustness of self-appraisal and enable monitoring of learning over time. ${ }^{28}$ The program is ongoing with a specific focus on exploring how the outcomes of the simulation program influence clinicians' practice and, ultimately, family experiences and rates of family decline to organ donation. Evaluation of the program in clinical practice is being undertaken in the COMFORT study (ClinicalTrials.gov (NCT01922310). ${ }^{29}$

\section{Conclusions}

We developed and delivered a highly effective and well-received simulation program that provided an opportunity to refine communication skills and techniques to increase the confidence of health professionals leading the donation conversation. Participants identified that skills learned in this program and the opportunity to rehearse conversations in realistic scenarios greatly enhanced their confidence. Overall, it is anticipated that this specialized and targeted training for DRs will contribute to enhanced donation conversations conducted by clinicians with 
greater skills and confidence, achieving improved family experiences of this difficult situation and subsequently increased consent rates for organ and tissue donation in NSW.

\section{Declaration of Conflicting Interests}

The author(s) declared no potential conflicts of interest with respect to the research, authorship, and/or publication of this article.

\section{Statement of Informed Consent}

Evaluation material provided data for ongoing review of teaching and content of the SP. The Human Research Ethics Committee (HREC) advised approval was not required for this educational activity. HREC advice was to notify participants of the use of their completed evaluation forms for publication and provide them an opportunity to decline use of their deidentified data. All participants had previously volunteered their names on the evaluation forms and investigators provided individuals with emailed information for informed consent. No participant declined use of his or her evaluation material included in the study. All participants signed a confidentiality agreement and consent for A-V recording before each workshop. Actors signed a confidentiality and media agreement annually. 
References

1. Siminoff LA, Marshall HM, Dumenci L, Bowen G, Swaminathan A, Gordon N. Communicating effectively about donation: an educational intervention to increase consent to donation. Progress in Transplantation. 2009;19(1):35-43.

2. Simpkin AL, Robertson LC, Barber VS, Young JD. Modifiable factors influencing relatives' decision to offer organ donation: systematic review. BMJ. 2009;338:b991.

3. Vincent A, Logan L. Consent for organ donation. British Journal of Anaesthesia. 2012;108 Suppl 1:i80-87.

4. Matesanz R, Dominguez-Gil B, Coll E, de la Rosa G, Marazuela R. Spanish experience as a leading country: what kind of measures were taken? Transplant International. 2011;24(4):333-343.

5. Shafer TJ, Ehrle RN, Davis KD, et al. Increasing organ recovery from level I trauma centers: the in-house coordinator intervention. Progress in Transplantation. 2004;14(3):250-263.

6. Australian Government. National Reform Agenda. 2008; http://www.donatelife.gov.au/about-us/national-reform-programme. Accessed 08 May, 2012.

7. $\quad$ Organ and Tissue Authority. Professional Education Package. 2014; http://www.donatelife.gov.au/professional-education-package. Accessed 18 October, 2016.

8. Mulvania P, Mehakovic E, Wise C, Cass Y, Daly TA, Nathan HM. Successful international collaboration improves family donation conversations resulting in increased organ donation. Transplantation Proceedings. 2014;46(6):2058-2065.

9. Australian and New Zealand Intensive Care Society. The ANZICS Statement on Death and Organ Donation. 3.2 ed. Melbourne: ANZICS; 2013: http://www.anzics.com.au/Pages/DaOD.aspx. Accessed 21 August 2015.

10. Organ and Tissue Authority. The Australasian Donor Awareness Program (ADAPT). 2015; http://www.donatelife.gov.au/adapt. Accessed 21 Aug, 2015.

11. Mullins GC, Simes D, Yuen KJ. Approaching families for organ donation-intensivists' perspectives. Anaesthesia and Intensive Care. 2012;40(6):1035-1039.

12. Pearson IY, Zurynski Y. A survey of personal and professional attitudes of intensivists to organ donation and transplantation. Anaesthesia and Intensive Care. 1995;23(1):68-74.

13. Thomas SL, Milnes S, Komesaroff PA. Understanding organ donation in the collaborative era: a qualitative study of staff and family experiences. Internal Medicine Journal. 2009;39(9):588-594.

14. NSW Ministry of Health. Increasing Organ Donation in NSW - Government Plan 2012. In: Coordination and Policy Unit, ed. North Sydney 2012.

15. Lave J, Wenger E. Situated learning: legitimate peripheral participation. Cambridge: Cambridge University Press; 1991.

16. Ellermann CR, Kataoka-Yahiro MR, Wong LC. Logic models used to enhance critical thinking. Journal of Nursing Education. 2006;45(6):220-227.

17. Hopwood N, Rooney D, Boud D, Kelly MA. Simulation in Higher Education: A sociomaterial view. Educational Philosophy and Theory. 2014. 
18. Schatzki T. A primer on practices. In: Higgs J, Barnett R, Billett S, Hutchings M, Trede F, eds. Practice-based education: Perspectives and strategies. Rotterdam: Sense; 2012:13-26.

19. Rudolph JW, Simon R, Rivard P, Dufresne RL, Raemer DB. Debriefing with good judgment: combining rigorous feedback with genuine inquiry. Anesthesiology Clinics. 2007;25(2):361-376.

20. Graneheim UH, Lundman B. Qualitative content analysis in nursing research: concepts, procedures and measures to achieve trustworthiness. Nurse Education Today. 2004;24(2):105-112.

21. Browning DM, Meyer EC, Truog RD, Solomon MZ. Difficult conversations in health care: cultivating relational learning to address the hidden curriculum. Academic Medicine. 2007;82(9):905-913.

22. Hales BM, Hawryluck L. An interactive educational workshop to improve end of life communication skills. Journal of Continuing Education in the Health Professions. 2008;28(4):241-248; quiz 249-255.

23. Meyer EC, Sellers DE, Browning DM, McGuffie K, Solomon MZ, Truog RD. Difficult conversations: improving communication skills and relational abilities in health care. Pediatric Critical Care Medicine. 2009;10(3):352-359.

24. Morton J, Blok GA, Reid C, van Dalen J, Morley M. The European Donor Hospital Education Programme (EDHEP): enhancing communication skills with bereaved relatives. Anaesthesia and Intensive Care. 2000;28(2):184-190.

25. van Dalen J, Blok GA, Morley MJ, et al. Participants' judgements of the European Donor Hospital Education Programme (EDHEP): an international comparison. Transplant International. 1999;12(3):182-187.

26. O'Regan S, Molloy E, Watterson L, Nestel D. Observer roles that optimise learning in healthcare simulation education: a systematic review. Advances in Simulation. 2016;1(4).

27. Rooney D, Hopwood N, Boud D, Kelly MA. The Role of Simulation in Pedagogies of Higher Education for the Health Professions: Through a Practice-Based Lens. Vocations and Learning. 2015:1-17.

28. Calhoun AW, Rider EA, Meyer EC, Lamiani G, Truog RD. Assessment of communication skills and self-appraisal in the simulated environment: feasibility of multirater feedback with gap analysis. Simulation in Healthcare. 2009;4(1):22-29.

29. COMmunication with Families regarding ORgan and Tissue Donation after Death in Intensive Care study. 2013. http://www.ANZCTR.org.au/ACTRN12613000815763.aspx. 
Appendix A.

\section{A case example: Donation after circulatory determination of death.}

"Sam" is a 24-year-old male with a large subdural hemorrhage and base of skull fracture

following an unprovoked assault that has received national media attention. Family members

present in the hospital are his parents and twin brother. The family is in shock by this unexpected and public event. The managing intensivist considers progression to brain death unlikely; therefore, donation after circulatory determination of death is to be discussed with the family. The family members have several concerns that need to be addressed before they will consent to donation. 
- 19 - 
Box 1. Workshop minimum personnel and resource requirements.

Personnel

- One workshop coordinator: to brief the actors, facilitate the timetable, welcome and organize participants.

- Professional actors experienced in playing patient roles and debriefing: roles of two family members.

- Managing team: roles of an intensivist (played by the facilitator), and a bedside nurse.

- Two participants (learner 'designated requesters').

- Two experts: a health professional facilitator and a subject expert in the FDC module content.

- Staff for scene setup and take down; sourcing appropriate props.

- One simulation technician to run the A-V system including playback; subsequent minor editing.

\section{Facilities and equipment}

- Separate area with a table for the facilitator to brief participants as a group at the beginning and debrief after the workshop.

- Separate area for actors to create backstories, rehearse and get into character in preparation for their roles.

- Simulation laboratory with a viewing room for the participant observer to watch the scenario in real time.

- Scenario props: a three-seat sofa, two armchairs, a coffee table, tissues, water and glasses.

- A-V system with essential features of real time viewing, replay, digital file copying facilities; optionally, editing with annotation.

Abbreviations: A-V, audio-visual; FDC, family donation conversation. 
Box 2. The debriefing process.

\begin{tabular}{ll}
\hline Debriefing & Activity \\
Part 1 Planning meeting & After the planning meeting, an informal discussion led by \\
debrief. & the facilitator, reflects on the team plan and conduct of the \\
& meeting. Any information missed by the participant is raised \\
& at this point. If the participant has not specified how they \\
& would like to be introduced to the family, this is established. \\
Part 2 End of the FDC & When the FDC is ended, the facilitator enters the room. \\
debrief. & Feedback is sought from the family in character, to garner \\
& initial reactions and emotions, and the participant is \\
& encouraged to question the family. Then, at the discretion of \\
& the facilitator, the family and nurse are directed to come out- \\
& of-character, and offer further feedback. Once the initial \\
debrief is complete the actors leave the room. & The observer and the subject matter expert enter the \\
debriefing area. A final facilitated debrief uses a video- \\
reflexive technique to trigger participants' insight and \\
reflection on practice. Annotations/notes on the A-V \\
recording are used as discussion points between the \\
facilitator, subject matter expert, observer and participant. \\
Standardized criteria are used to guide achievement of key \\
learnings from the national FDC workshops. A digital file of \\
the video recording is provided for the participant's personal \\
ongoing reflection.
\end{tabular}

Abbreviations: A-V, audio-visual; FDC, family donation conversation. 
Table 1. Characteristics of workshop evaluation respondents.

\section{Characteristic}

Female, No. (\%)

Intensivist, No. (\%)

Intensive care nurses and social worker ${ }^{\mathrm{a}}$, No. (\%)

Attended workshop on one occasion, No. (\%)

Attended workshop twice, No. (\%)

Attended workshops three or more times, No. (\%)
Total $(\mathbf{n}=\mathbf{8 2})$

$41(50.0)$

$44(53.7)$

$38(46.3)$

$66(80.5)$

$13(15.9)$

$3(3.6)$

${ }^{\mathrm{a}}$ Only one social worker attended. 
Table 2. Categories of elements liked most in the simulation workshop.

Category and subcategory

Count $^{\mathbf{a}}$

Feedback: person providing it; quality; topic; debriefing; video

playback

Use of professional actors

Realism/high fidelity scenarios

44

Opportunity to practice/usefulness

Setting: organization; safe learning environment

${ }^{a}$ Count equals the number of times referred to. 
Table 3. Categories of elements liked least in the simulation workshop.

Category and subcategory

Nothing

Performance anxiety

Being watched by self and others

Setting: practical considerations

Feedback

Actors

Processes

${ }^{\mathrm{a}}$ Count equals the number of times referred to.
Count $^{\mathrm{a}}$

22

13

12

12

3

2

2 